\title{
Intragranular Residual Stress Evaluation Using the Semi-Destructive FIB-DIC Ring-Core Drilling Method
}

\author{
Alexander J. G. Lunt ${ }^{1, a^{*}}$ and Alexander M. Korsunsky ${ }^{1, b}$ \\ ${ }^{1}$ Department of Engineering Science, University of Oxford, Parks Road, Oxford, OX1 3PJ, U.K. \\ aalexander.lunt@eng.ox.ac.uk, balexander.korsunsky@eng.ox.ac.uk
}

Keywords: Residual stresses, FIB, SEM, DIC, TiAl, Intragranular

\begin{abstract}
Titanium aluminide (TiAl) is a lightweight intermetallic compound with a range of exceptional mid-to-high temperature mechanical properties. These characteristics have the potential to deliver significant weight savings in aero engine components. However, the relatively low ductility of TiAl requires improved understanding of the relationship between manufacturing processes and residual stresses in order to expand the use of such components in service. Previous studies have suggested that stress determination at high spatial resolution is necessary to achieve better insight. The present paper reports progress beyond the current state-of-the-art towards the identification of the near-surface intragranular residual stress state in cast and ground $\mathrm{TiAl}$ at a resolution better than $5 \mu \mathrm{m}$.

The semi-destructive ring-core drilling method using Focused Ion Beam (FIB) and Digital Image Correlation (DIC) was used for in-plane residual stress estimation in ten grains at the sample surface. The nature of the locally observed strain reliefs suggests that tensile residual stresses may have been induced in some grains by the unidirectional grinding process applied to the surface.
\end{abstract}

\section{Introduction}

The titanium aluminide intermetallic is a lightweight, high temperature material consisting one or more of the three main compounds; $\gamma \mathrm{TiAl}, \alpha_{2} \mathrm{Ti}_{3} \mathrm{Al}$, and $\mathrm{TiAl}_{3}$. These intermetallics demonstrate high temperature strength and stiffness along with good creep and fatigue resistance [1]. These thermal properties, in combination with the relatively low density of TiAl $\left(\approx 4.0 \mathrm{gcm}^{-2}\right)$ have ensured that TiAl is finding more widespread use in mid-to-high temperature aeronautical applications.

Titanium aluminide components may be manufactured by conventional processes such as near net shape casting followed by machining operations to achieve the required tolerances at critical locations. However, such methods may induce undesirable near-surface stresses during manufacture and previous studies have demonstrated that the material integrity is particularly sensitive to the nature of this process and resulting residual stress at the surface [2]. For this reason, unidirectional grinding has been proposed as a manufacturing process that allows both shape control and compressive stress generation. Nevertheless, the effect of grinding remains insufficiently understood and further analysis is required.

Measuring Residual Surface Stresses. The nature of external force application means that near-surface residual stresses are often the most influential in terms of structural integrity. For this reason, a suite of techniques have been developed to quantify such behaviour at both the macro and micro-scale.

Hole drilling is a technique based on the strain relief associated with the traction free surfaces of a hole. Strains are quantified using strain gauges or DIC [3], and then used to calculate the residual stress at the hole and the surrounding region. The characteristic length scale of this strain relief means that this technique can only quantify average stresses, or intragranular stresses in coarse-grained materials.

An alternative approach to measuring surface residual stress is the use of X-ray Diffraction (XRD) methods. Such techniques exploit the interaction between incident X-rays and the crystallographic lattice planes to determine the interatomic strains present in a material. Traditional techniques such as the $\sin ^{2} \psi$ method are based on averaging many thousands of grains and 
therefore are unsuitable for calculating stresses in individual grains. An alternative approach, based on recent advancements in XRD micro-focusing has enabled a limited number of XRD synchrotron facilities to quantify elastic strains in individual grains using the analysis of Laue diffraction patterns [4].

The introduction of traction-free surfaces using FIB milling, and the measurement of the induced strain changes using DIC is a new technique which can be used to determine the residual stress at the micro/nano-scale. One approach involves the use of linear slots or holes in a manner similar to macro-scale hole drilling [5] however material independent usage of this method has proven to be difficult.

An alternative FIB and DIC approach involves stress relieving an island of material at the specimen surface. This technique, also known as the micro-ring-core method, is based on repeated SEM imaging of a central core in order to quantify surface strain variation as a function of the cut depth. A value for the average in-plane residual stress in the core region can then be determined by comparison with finite element results. This technique has been successfully applied to polycrystalline materials at the micro/nano scale [6,7] and offers a resolution sufficiently high to quantify intragranular strains in microstructured materials of practical significance.

At this stage it is important to highlight the fact that the existing ring-core interpretation approaches are based on isotropic, continuum mechanics approximations. Thus, in the case of elastically anisotropic crystals, only the tensile or compressive nature of the pre-existing stress can still be determined (which is opposite in sign to the strain relief observed during milling). Without knowledge of the crystal orientation, any stress quantification can only ever serve as an estimate. The analysis of the surface strain relief is nevertheless helpful in determining the influence of grinding and grain orientation on the residual stress state. This paper aims to advance the current understanding of such effects and their influence on the structural integrity of components made from this material.

\section{Experimental Method}
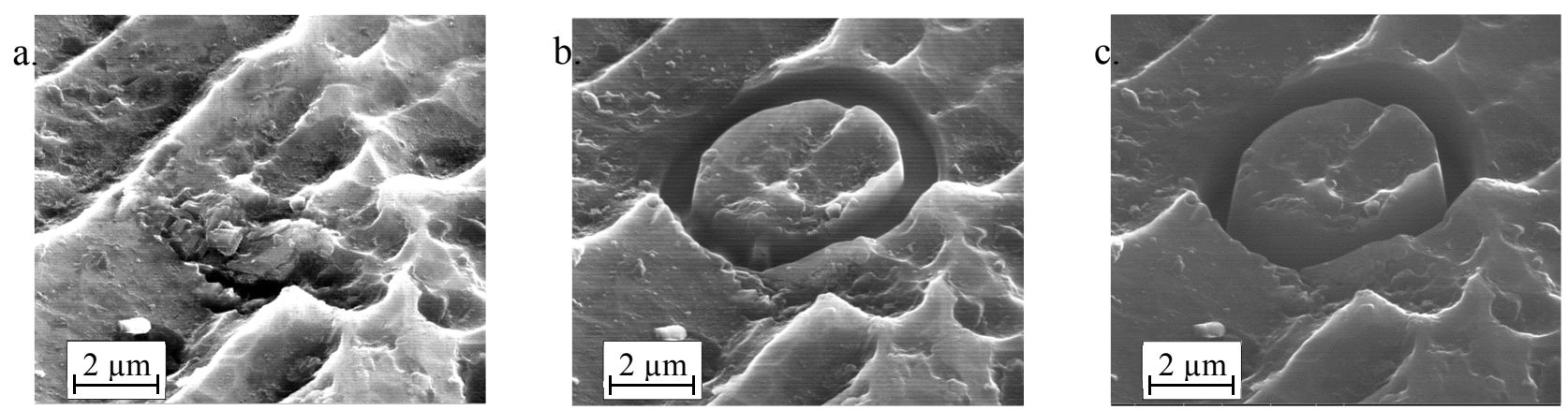

Fig 1: Micrographs of the stages of a typical ring-core milling process. a) Image of pristine surface.

b) Intermediate milling step. c) Final cut depth showing contrast reduction from material redeposition.

Material and Microstructure. TiAl intermetallic of the composition 45-48 wt $\%$ aluminium was centrifugally cast in a near-net-shape casting process and unidirectional grinding was applied to the surface. Sample coupons of size $10 \times 7 \times 4 \mathrm{~mm}^{3}$ were then cut for further analysis.

Micrographs of the sample surface were obtained as shown in Fig 1. A complex microstructure was observed with a majority phase $(80-90 \%)$ of $\gamma$ TiAl encapsulating $\alpha_{2} \mathrm{Ti}_{3} \mathrm{Al}$ lamella. Lamella orientation was found to be randomly distributed and this property was used to distinguish between individual grains. The nominal size of the grains was found to be between 70 and $100 \mu \mathrm{m}$.

Dual Beam SEM and FIB. The sample was placed into a FEI Scios DualBeam FIB/SEM chamber and ten random grains in a neighbourhood cluster were selected for further analysis.

Initial testing was performed to refine the milling setup of the FEB/SEM procedure. A large number of interrelated effects need to be considered when optimising the milling setup. This includes the stub diameter, ring-core width, milling rate and beam voltage. These parameters influence the resolution of the technique and the effect of noise (and re-deposition of cut material onto the stub surface (Fig. 1c)). 
Two differing cutting methodologies were initially tested on grains. The first relied on incremental milling followed by high resolution imaging to produce a smaller number of images with reduced noise component in each image. This process was implemented on grains 1-3. This approach was found to be very slow and resulted in a large amount of charging and beam drift. The alternative approach relied on continuous milling and imaging of the sample surface. Although the signal-to-noise ratio each image was found to be smaller in this approach, the increased number of images enabled effective strain profile fitting. This approach was used for the remainder of the grains.

Following this testing procedure, a $5 \mu \mathrm{m}$ core diameter and a trench width of $1 \mu \mathrm{m}$ was selected. Each imaging sequence captured over 300 images and took less than 15 minutes to implement.

Digital Image Correlation. Digital image correlation is a non-contact scale independent method of measuring strain. This technique relies upon initially seeding of the sample surface (Fig. 2c) followed by $2 \mathrm{D}$ tracking of the marker position using the correlation function.

Deposition of a surface layer followed by surface patterning is conventionally used to introduce surface contrast for DIC in ring-core FIB stress analysis [6, 7]. However, as shown in Fig.1 micrographs of the substrate surface show high levels of contrast and non-uniformity. DIC strain fitting on this surface was found to be highly effective (Fig. 2) and therefore no patterning was implemented.

a.

b.

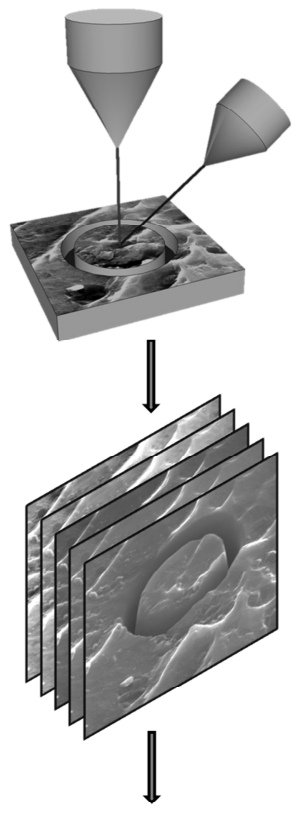

c.

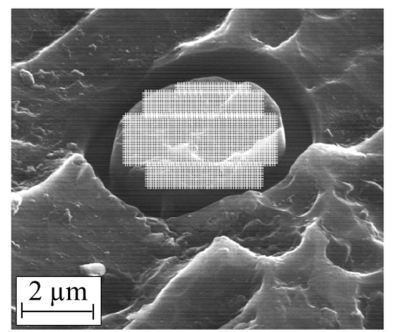

d3.

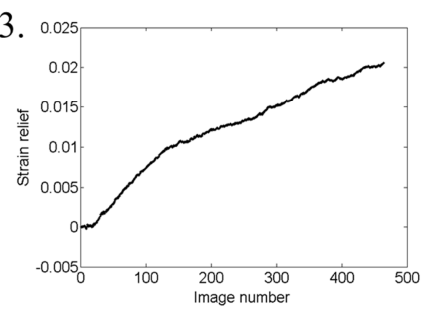

$\mathrm{d} 2$.

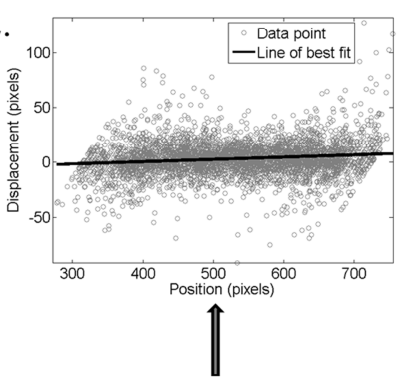

d1.

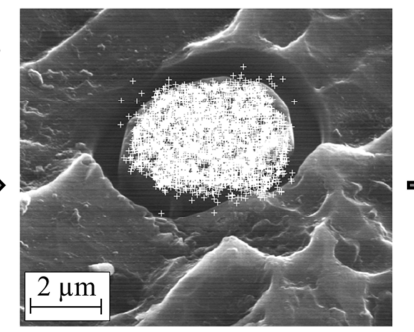

e3.

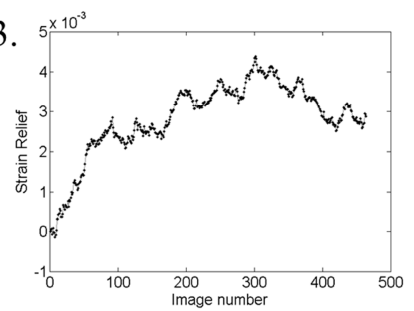

e2.

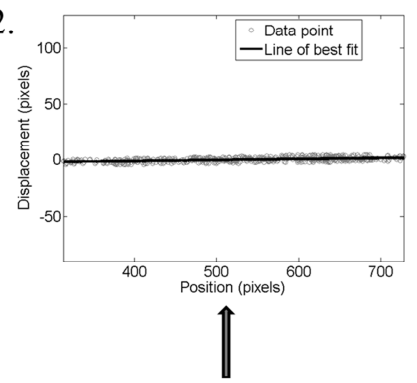

e1.

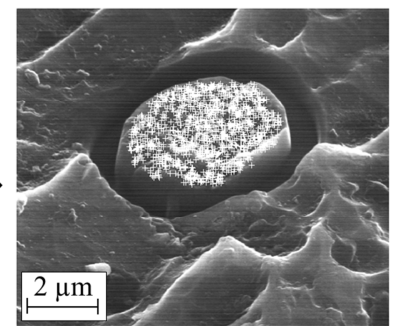

Fig 2. Schematic illustration of the steps of strain relief profile analysis. a) Simultaneous FIB milling (normal to sample surface) and SEM imaging (tilted at $52^{\circ}$ ). b) Image collection. c) Marker seeding on the central island region. d) Automated DIC showing large numbers of outliers. e) Removal of outliers and retention of well-tracked markers allows clear trends to be revealed in the displacement against pixel plot. The gradient of this plot is used to quantify strain relief as a function of image number.

A Matlab DIC code was used to perform strain quantification on the central stub region. Seeding of the core surface was performed by applying multiple markers (Fig. 2c) followed by automated DIC. Many poorly tracked markers were observed (Fig. 2d) and a procedure for outlier removal wasimplemented. Plots of marker displacement against pixel position were then used to find the 
gradient of displacement across the stub (the average strain) as a function of image number (Fig. $2 \mathrm{e} 2$ and $2 \mathrm{e} 3$ ). The standard deviation of this fitting process was also used as a measure of strain error.

The strain measurement approach implemented is only able to quantify strain in a single orientation during one implementation and horizontal strain analysis was performed on each image set. Limitations on imaging angle and electron scan directions mean that the noise component associated with the vertical strain relief is much larger. Therefore, in order to quantify strain relief in directions parallel and perpendicular to the grinding direction, the sample was rotated by $90^{\circ}$ and a second milling procedure was performed in each grain. The distance $(\approx 15 \mu \mathrm{m})$ between the markers in each of these implementations was selected as a compromise between avoiding the mutual influence of successive measurements, and making sure that the underlying stress state was consistent between the positions.

Profile Fitting. Direct comparison of the strain relief profile with a known strain profile is then used to determine the average full strain relief $\Delta \varepsilon_{\infty}$ (at infinite cut depth) at the ring-core surface [6]. The strain relief profile is independent of material properties and is based on a function of the cut depth $h$ and the ring-core diameter $d$ such that $z=(h / 0.42 d)$ :

$$
f\left(\Delta \varepsilon_{\infty}, z\right)=1.12 \Delta \varepsilon_{\infty} \times \frac{z}{(1+z)}\left[1+\frac{2}{\left(1+z^{2}\right)}\right] .
$$

Since the exact cut depth at a given image number is unknown, a parameter $\left(\kappa_{\text {mill }}\right)$ describing this relationship was introduced such that $h=I \kappa_{\text {mill }}$, where $I$ is the image number. An offset parameter $\left(\delta_{\text {start }}\right)$ was also introduced into the expression for $z$ to account for initial surface roughness such that:

$$
z=\frac{I \kappa_{\text {mill }}}{0.42 d}-\delta_{\text {start }}
$$

A least squares approach was then used to optimise the values of $\Delta \varepsilon_{\infty}, \kappa_{\text {mill }}$ and $\delta_{\text {start }}$ in each grain. The standard deviations of these parameters were also calculated from the variance of the strain values.

a.

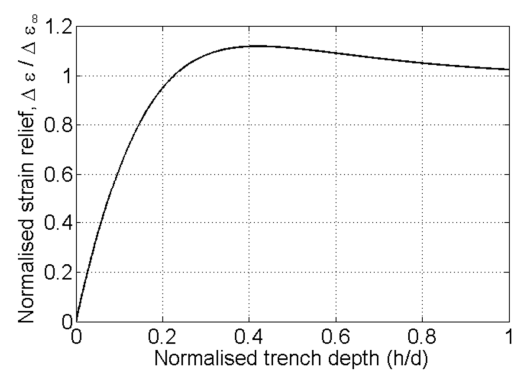

b.

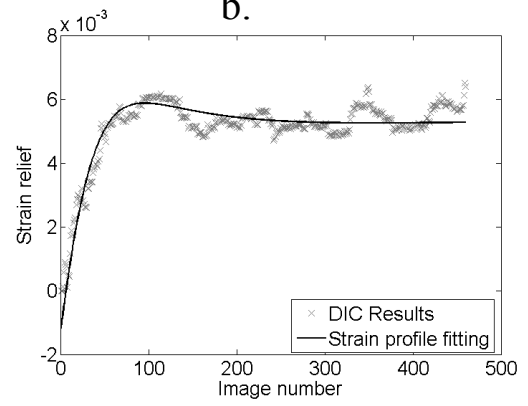

c.

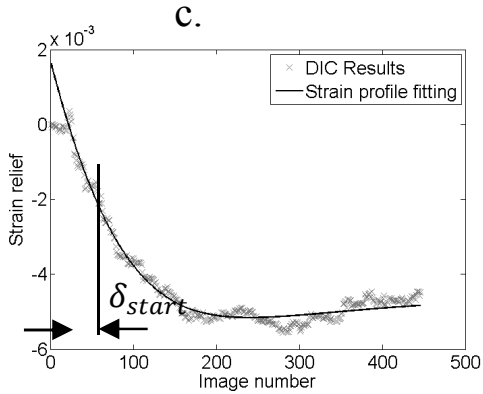

Fig. 3: Strain relief profile fitting. a) Normalised stress relief fitting against normalised drilling depth. b) Fitting of strain relief in grain 1 in a direction parallel to grinding. c) Fitting of strain relief

in grain 3 in a direction parallel to grinding direction with the shifting effect introduced by the offset parameter.

\section{Experimental Results}

Strain relief measurements were performed in continuous milling and imaging mode for the ten grains in directions parallel and perpendicular to the grinding direction. To allow direct comparison between different techniques, the results of the stepwise milling process in grains 1-3 were also included (Fig. 4a). 
The single crystals (or laminar oligocrystals) that constitute the grains in the $\mathrm{TiAl}$ and $\mathrm{Ti}_{3} \mathrm{Al}$ microstructure [8] clearly have anisotropic elastic properties. We observe, however, that the fitting
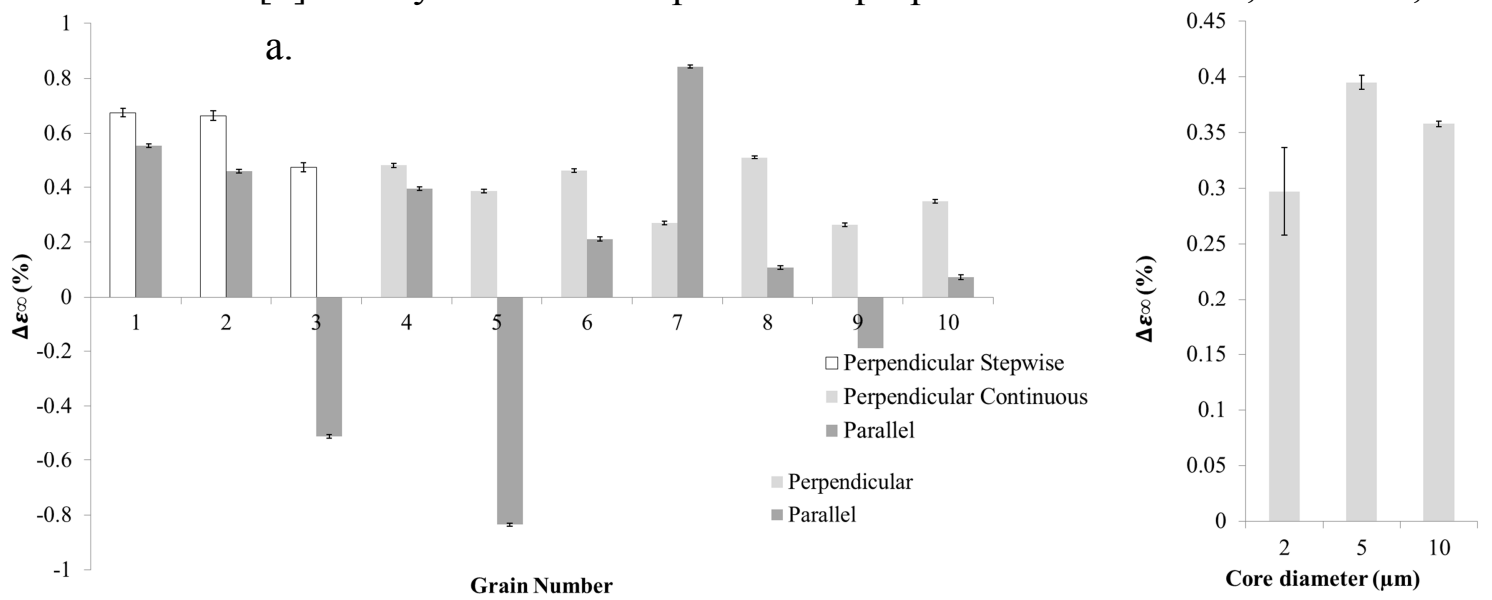

b.

Fig. 4 a) Full strain relief against grain number in directions parallel and perpendicular to the grinding direction for both the stepwise and continuous milling process. b) Full strain relief against core diameter size in grain 4 . Error bars indicate the $99 \%$ confidence intervals on both plots.

errors between the predicted isotropic strain relief curve and the measured strain profiles remained small throughout. This suggests that the anisotropic crystal behaviour has little influence on the redistribution of strain during ring-core milling.

In order to determine the influence of resolution on residual strain measurement, the process was also repeated in grain 4 in a direction parallel to the grinding direction using two further core diameters ( 2 and $10 \mu \mathrm{m})$ as shown in Fig. $4 \mathrm{~b}$.

The compressive or tensile nature of the residual in-plane stress state in a given direction can then be unequivocally determined from the sign of the ring-core strain relief. Positive strain relief is indicative of compressive stress, and negative strain relief corresponds to a pre-existing tensile stress.

\section{Discussion}

Ten Grain Results. The estimates of the full strain relief in each grain are shown in Fig. 4a. The positive strain relief values observed confirm that most grains were found to be in a state of compression in both orientations (parallel and perpendicular to the grinding direction). Exceptions were grains numbered 3,5 and 9 which demonstrated negative strain relief sign, and thus a tensile residual stress. Since in all cases this was associated with the orientation along the grinding direction, there appears to exist a correlation between the grinding process and residual stress generation. In other respects, the relief strains did not appear to be correlated with any of the local features, parameters such as $\delta_{\text {start }}, \kappa_{\text {mill }}$ or the in-plane lamella orientation. Rogue grain interaction has the potential to exert strong influence on structural integrity, as outlined by Dunne et al. [9]. However, establishing such correlation requires crystal orientation analysis. The identification of grain Euler angle was not performed as part of this study.

Examination of the $99 \%$ confidence intervals in Fig. 4a shows that the error level in the stepwise milling process is significantly larger than in the continuous procedure. Although the amount of noise per image is larger in the continuous milling process, the increased number of images is sufficient to reduce the overall error.

Ring-Core Diameter Analysis. The full strain relief against ring-core diameter in grain 4 is shown in Fig. 4b. In-grain variation of this strain response is expected and the measurements were performed at $15 \mu \mathrm{m}$ intervals. Despite this, these measurements are consistent to within $16 \%$ suggesting that the implementation of the ring-core process is not dependent upon core diameter. The error associated with strain measurements is however dependent upon core diameter and an 
inverse relationship between the ring-core cross sectional area and the $99 \%$ confidence intervals can be demonstrated.

\section{Conclusion}

This paper outlines the implementation of the ring-core FIB and DIC surface residual stress evaluation technique to a ground surface of TiAl. The technique was applied to determine the residual strain in 10 grains at the resolution of $5 \mu \mathrm{m}$. It was found that most grains exhibited a compressive residual stress in directions both parallel and perpendicular to the grinding direction. However, for three grains, the analysis revealed tensile stress in the orientation along the grinding direction. This suggests that further control over the processing conditions for unidirectional grinding must be exercised in order to ensure entirely compressive near-surface stress state in the material.

Analysis into the effect of ring-core diameter on the residual strain was also performed by implementing the ring-core FIB-DIC analysis with the core diameters of 2,5 and $10 \mu \mathrm{m}$ within a single grain. These results were found to be consistent to within $16 \%$, suggesting that the application of the approach is able to provide reliable estimates of the relief strain in the range of length scales covered.

In order to quantify estimates of the intragranular stress, further work on the influence of anisotropic single crystal response on the ring core FIB-DIC approach needs to be performed. This requires re-implementation of the FE ring-core model in which grain orientation angle and the corresponding anisotropic stiffness matrix is modelled. The identification of single crystal orientation and of the principal strains would then need to be carried out as part of the experimental characterisation.

\section{References}

[1] M. Goral, et al., Diffusion aluminide coatings for TiAl intermetallic turbine blades, Intermetallics. 19(5) (2011) 744-747.

[2] A.T. Yokobori, et al., The characterization of creep crack growth rate and its life of TiAl intermetallic compound with full lamellar microstructure, INT J PRES VES PIP. 78(11-12) (2001) 757-764.

[3] G.S. Schajer, Advances in Hole-Drilling Residual Stress Measurements, Experimental Mechanics. 50(2) (2010) 159-168.

[4] F. Hofmann, et al., Analysis of strain error sources in micro-beam Laue diffraction, NUCL INSTRUM METH A. 660(1) (2011) 130-137.

[5] M. Krottenthaler, et al., A simple method for residual stress measurements in thin films by means of focused ion beam milling and digital image correlation, SURF COAT TECH. 215 (2013) 247-252.

[6] A.M. Korsunsky, M. Sebastiani and E. Bemporad, Focused ion beam ring drilling for residual stress evaluation, Materials Letters. 63(22) (2009) 1961-1963.

[7] M. Sebastiani, et al., Depth-resolved residual stress analysis of thin coatings by a new FIB-DIC method, MAT SCI ENG A-STRUCT. 528(27) (2011) 7901-7908.

[8] K. Tanaka and M. Koiwa, Single-crystal elastic constants of intermetallic compounds, Intermetallics. 4 (1996) 29-39.

[9] F.P.E. Dunne, A. Walker and D. Rugg, A systematic study of hep crystal orientation and morphology effects in polycrystal deformation and fatigue, P R SOC A. 463(2082) (2007) 1467-1489. 